\title{
Family history of heart disease and cardiovascular disease risk-reducing behaviors
}

\author{
Margaret E. McCusker, MD, MS $S^{1}$, Paula W. Yoon, ScD, MPH ${ }^{2}$, Marta Gwinn, MD, MPH ${ }^{2}$,
} Ann M. Malarcher, PhD, MSPH ${ }^{3}$, Linda Neff, $P h D^{3}$, and Muin J. Khoury, $M D, P h D^{2}$

\begin{abstract}
Background: Family history is an important cardiovascular disease (CVD) risk factor. Preventive behaviors, including lifestyle modifications, can attenuate CVD risk. We studied the association between family history-based heart disease (HD) risk and CVD risk-reducing behaviors. Methods: Using data from the 2001 Healthstyles survey, we compared frequencies of CVD risk-reducing behaviors among adults without known CVD in categories defined by family historybased HD risk. We classified respondents' HD risk as average (no first-degree relatives with HD), moderate (one relative), or high ( $\geq$ two relatives). Behaviors studied included lifestyle modifications, cholesterol measurement, and aspirin use. Results: Of 3383 respondents without known CVD, 28\% were classified as being at moderate risk and 15\% as being at high risk for HD based on family history. Adjusted odds ratios indicated that moderate- and high-risk respondents were more likely to report having cholesterol measured within the previous 5 years $(\mathrm{OR}=1.39,95 \%$ Confidence Interval $[\mathrm{Cl}]=1.16-1.67$ and $1.29,95 \% \mathrm{Cl}=1.01-1.64$, respectively), and aspirin use to reduce $\mathrm{CVD}$ risk $(\mathrm{OR}=1.49,95 \% \mathrm{Cl}=1.23-1.79$ and $1.67,95 \% \mathrm{Cl}=1.33-2.09$, respectively) than average-risk respondents. Conclusion: Almost one half of respondents reported a family history of HD. Aspirin use and cholesterol measurement (i.e., behaviors that health-care providers might suggest) were more likely to be reported by moderate- and high-risk respondents than were lifestyle changes. Family history merits further investigation as a public health tool to identify persons with increased HD risk who might benefit from enhanced prevention strategies. Genet Med 2004:6(3):153-158.
\end{abstract}

Key Words: cardiovascular disease, family history, risk, heart disease, behavior

Cardiovascular disease (CVD) is the leading cause of death in the United States, with over 700,000 deaths attributable to diseases of the heart and almost 170,000 deaths attributable to cerebrovascular diseases in $2000 .{ }^{1}$ In addition to the substantial loss of life caused by these diseases, the financial costs of CVD for health care and lost productivity were estimated to exceed $\$ 350$ billion in 2003. ${ }^{2}$

Family history, a well-known risk factor for CVD, represents genetic, environmental, and behavioral elements, and the interactions between them. ${ }^{3}$ For heart disease (HD), the relative risk ranges from 2.0 to 9.0 among persons who report a family history of HD, depending on the type and number of relatives considered. ${ }^{4}$ For stroke, the relative risk ranges from 1.5 to 2.0 among first-degree relatives of persons who have had a stroke. ${ }^{5,6}$ Based on

\footnotetext{
${ }^{1}$ Epidemic Intelligence Service assigned to the Texas Department of Health, Epidemiology Program Office; ${ }^{2}$ Office of Genomics and Disease Prevention, National Center for Environmental Health; and ${ }^{3}$ Cardiovascular Health Branch, Division of Adult and Community Health, National Center for Chronic Disease Prevention and Health Promotion, Centers for Disease Control and Prevention, Atlanta, Georgia.

Margaret E. McCusker, MD, MS, Texas Department of Health, Bureau of Chronic Disease and Tobacco Prevention, 1100 West 49th Street, T-402, Austin, TX 78756.

Received: November 4, 2003

Accepted: January 15, 2004.
}

DOI: 10.1097/01.GIM.0000127271.60548.89 these associations, family history can be used to identify a group of people who have above-average risk for CVD. ${ }^{7}$

Family history may be used as a tool to educate persons about their risk and encourage behaviors that can reduce the risk of CVD. ${ }^{8,9}$ Modifiable CVD risk factors include hypertension, hypercholesterolemia, physical inactivity, overweight/obesity, and tobacco use., $, 5,10$ Modification of these risk factors through changes in diet, increased physical activity, tobacco use cessation, and pharmacotherapy (when needed) can reduce CVD risk considerably for the majority of the population. ${ }^{11}$ Persons who are at high risk for CVD because of inherited single-gene disorders such as familial hypercholesterolemia can be referred for extensive clinical evaluation and management.

Adults who report that one or more first-degree relatives has HD might be more likely to practice CVD risk-reducing behaviors than adults who do not report any first-degree relatives with HD. ${ }^{4}$ To evaluate the connection between family history and behavior among adults without known CVD, we studied the association between family history-based HD risk and risk-reducing behaviors in a national survey.

\section{MATERIALS AND METHODS}

\section{Study design}

We selected subjects for this cross-sectional study from respondents to the 2001 Healthstyles survey. Healthstyles is a 
national mail survey of health attitudes, behaviors, conditions, and knowledge. ${ }^{12}$ The survey has been conducted annually since 1995 for Porter-Novelli, a social marketing and public relations firm. Market Facts, Inc., administers the Healthstyles survey to all respondents to the DDB Needham Lifestyles survey. ${ }^{12,13}$ For the Lifestyles survey, a stratified random sample is drawn from a panel of volunteers who have been recruited to participate in market research. The strata are based on eight factors (age, sex, marital status, race/ethnicity, income, geographic region, household size, and population density) to create a sample representative of the U.S. adult population. Additional surveys are mailed to low-income and minority households to improve representation of these groups among Lifestyles respondents. For the 2001 Healthstyles survey, weights based on U.S. census figures for five factors (age, sex, race/ethnicity, income, and household size) were developed after stratification. Several health agencies, including the Centers for Disease Control and Prevention, assist with question development for the Healthstyles survey.

\section{Variable definitions}

The 2001 Healthstyles survey included a series of four questions regarding family history, which asked respondents if their biological mother, father, brothers, or sisters (first-degree relatives) ever had "asthma" or "heart disease." Because the survey questions did not require respondents to specify the age of heart disease onset, we used a modified version of the guidelines developed by Scheuner et al ${ }^{14}$ to classify individuals into three HD risk categories according to the number of affected first-degree relatives they reported. We classified respondents who reported that no first-degree relatives had HD or who did not know if any first-degree relatives had HD as at average risk for HD; those who reported one first-degree relative with HD as moderate risk, and those who reported two or more firstdegree relatives with HD as high risk.

The 2001 Healthstyles survey also included questions about behaviors that can reduce the risk of developing CVD. Respondents were asked if they practiced any of the following behaviors to lower their risk of developing HD or stroke: eating fewer high fat or high cholesterol foods, eating more fruits and vegetables, being more physically active, or trying to stop smoking. In addition to these questions, respondents were asked if they used aspirin to reduce the chance of a heart attack or stroke, and when they last had their blood cholesterol measured. We classified respondents as having hypertension or hypercholesterolemia if they reported that a health care professional had ever told them that they had the condition. We classified respondents as having diabetes if they indicated that they had the disease on a checklist of health conditions. Respondents who reported that they smoked at least one cigarette per day were classified as current cigarette smokers. We defined overweight/obesity as a body mass index of $25 \mathrm{~kg} / \mathrm{m}^{2}$ or greater based on self-reported height and weight.

\section{Statistical analysis}

We compared demographic characteristics, CVD risk factors, and CVD risk-reducing behaviors among the three family history-based HD risk categories using chi-square tests and tests for trend. We evaluated associations between demographic characteristics that varied significantly among the three risk categories and CVD risk-reducing behaviors to look for interactions. We conducted age-stratified analyses to examine the association of CVD risk-reducing behaviors with both family history-based HD risk and a report of at least one sibling with HD. We created logistic regression models for each of the CVD risk-reducing behaviors, adjusting for family history-based HD risk, demographic characteristics, and whether or not respondents visited a primary care physician within the previous year. We performed all analyses with and without the weights that were developed for the Healthstyles data and found only minor differences between the weighted and unweighted results. Hence, we present the unweighted results. Statistical significance was set at a two-sided alpha of 0.05 . Odds ratios are reported with $95 \%$ confidence intervals. We used SAS version 8.2 (SAS Institute, Cary, North Carolina, 2001) to perform all analyses.

\section{RESULTS}

During 2001, the Healthstyles questionnaire was mailed to 5605 U.S. adults who responded to the Lifestyles survey (Lifestyles response rate: 64\%); 66\% (3719) returned the Healthstyles questionnaires. Compared to 2000 U.S. census estimates, the Healthstyles sample included higher proportions of females, persons aged 35-54 years, college graduates, and married persons. ${ }^{15}$

We included the 3383 respondents without known CVD in our study. These individuals indicated that they had no history of stroke, heart attack, angina, or coronary heart disease on the Healthstyles questionnaire. We excluded 10 respondents with an unknown history of CVD. Of the CVD-free respondents, $57 \%$ reported family history information that placed them in the average HD risk category; $28 \%$ and $15 \%$ were classified as at moderate and high risk for HD, respectively. Eighty (2\%) of the respondents did not know whether any of their first-degree relatives had $\mathrm{HD}$ and were classified as at average risk for HD. There were 370 respondents who reported having at least one sibling with HD. Of all respondents, $86 \%$ reported at least one visit to a primary-care physician within the previous 12 months.

The demographic characteristics and CVD risk factors among respondents in the three risk categories are included in this report (Table 1). The proportions of respondents aged 45 years and older increased with family history-based HD risk. The moderate- and high-risk categories contained slightly higher proportions of respondents who classified themselves as white. Except for current cigarette smoking, the proportion of respondents who reported having any of the CVD risk factors increased with family history-based HD risk. Regardless 
Table 1

Description of respondents classified by family history-based heart disease risk $(N=3383)$

\begin{tabular}{|c|c|c|c|c|c|c|c|}
\hline \multirow[b]{2}{*}{ Demographics } & \multicolumn{2}{|c|}{ Average risk ${ }^{a}$} & \multicolumn{2}{|c|}{ Moderate risk ${ }^{b}$} & \multicolumn{2}{|c|}{ High risk ${ }^{c}$} & \multirow[b]{2}{*}{$P^{d}$} \\
\hline & $N$ & $(\%)$ & $N$ & $(\%)$ & $N$ & $(\%)$ & \\
\hline Total & 1913 & $(100)$ & 953 & (100) & 517 & (100) & NA \\
\hline Age, years & & & & & & & $<0.01$ \\
\hline $18-34$ & 476 & (25) & 95 & (10) & 28 & (5) & \\
\hline $35-44$ & 711 & $(37)$ & 348 & $(36)$ & 116 & $(22)$ & \\
\hline $45-64$ & 577 & $(30)$ & 388 & $(41)$ & 247 & $(48)$ & \\
\hline$\geq 65$ & 149 & (8) & 122 & (13) & 126 & (25) & \\
\hline Sex & & & & & & & 0.06 \\
\hline Male & 733 & $(38)$ & 327 & (34) & 182 & (35) & \\
\hline Female & 1180 & $(62)$ & 626 & $(66)$ & 335 & $(65)$ & \\
\hline Race/Ethnicity & & & & & & & $<0.01$ \\
\hline White & 1403 & (73) & 747 & (78) & 403 & (78) & \\
\hline Black & 228 & (12) & 92 & (10) & 58 & (11) & \\
\hline Hispanic & 195 & (10) & 86 & (9) & 39 & (8) & \\
\hline Other & 87 & (5) & 28 & (3) & 17 & (3) & \\
\hline Education $^{e}$ & & & & & & & 0.08 \\
\hline High school grad or less & 617 & $(33)$ & 298 & $(31)$ & 210 & $(41)$ & \\
\hline Some college & 686 & (37) & 338 & $(36)$ & 161 & $(31)$ & \\
\hline College grad or more & 567 & $(30)$ & 308 & $(33)$ & 143 & (28) & \\
\hline Income & & & & & & & 0.61 \\
\hline$<\$ 25,000$ & 440 & $(23)$ & 219 & $(23)$ & 137 & $(26)$ & \\
\hline$\$ 25,000-59,999$ & 773 & $(40)$ & 347 & $(36)$ & 196 & $(38)$ & \\
\hline$>\$ 59,999$ & 700 & (37) & 387 & $(41)$ & 184 & $(36)$ & \\
\hline \multicolumn{8}{|l|}{ CVD Risk Factors } \\
\hline Hypertension & 418 & $(22)$ & 282 & $(30)$ & 225 & $(44)$ & $<0.01$ \\
\hline Hypercholesterolemia & 439 & (23) & 316 & $(33)$ & 211 & $(41)$ & $<0.01$ \\
\hline Diabetes & 146 & (8) & 98 & $(10)$ & 74 & (14) & $<0.01$ \\
\hline Current cigarette smoker & 406 & $(21)$ & 207 & $(22)$ & 115 & $(22)$ & 0.59 \\
\hline Overweight/Obese $^{f}$ & 1117 & $(58)$ & 601 & (63) & 355 & (69) & $<0.01$ \\
\hline
\end{tabular}

${ }^{a}$ Chi-square test for trend.

${ }^{b}$ Average risk defined as no first-degree relatives known to have heart disease.

${ }^{c}$ Moderate risk defined as one first-degree relative with heart disease.

${ }^{d}$ High risk defined as two or more first-degree relatives with heart disease.

${ }^{e}$ There were 3328 people who supplied information about education.

${ }^{f}$ Body mass index $\geq 25 \mathrm{~kg} / \mathrm{m}^{2}$.

CVD, cardiovascular disease.

of HD risk category, respondents aged 45 years and older were almost twice as likely to have hypertension, hypercholesterolemia, and diabetes compared with respondents aged 18-44 years.

Most respondents reported practicing CVD risk-reducing behaviors, regardless of family history-based HD risk category (Table 2). Tests for trend showed significant increases associated with increased HD risk in the proportion of respondents who reported eating fewer high-fat foods $(P<0.01)$, eating more fruits and vegetables $(P<0.01)$, having a serum cholesterol checked within the previous 5 years $(P<0.01)$, and using aspirin to reduce CVD risk $(P<0.01)$. However, when we controlled for age, only the increases in serum cholesterol measurement $(P<0.01)$ and aspirin use $(P=0.02)$ remained significantly associated with increasing family history-based HD risk.

Adjusted odds ratios for the likelihood of reporting CVD risk-reducing behaviors among moderate and high-risk re- 
Table 2

CVD risk-reducing behaviors among respondents classified by family history-based heart disease risk

\begin{tabular}{|c|c|c|c|c|c|c|}
\hline \multirow[b]{2}{*}{ Behavior $^{d}$} & \multicolumn{2}{|c|}{ Average risk ${ }^{a}$} & \multicolumn{2}{|c|}{ Moderate risk $^{b}$} & \multicolumn{2}{|c|}{ High risk $^{c}$} \\
\hline & $N$ & $(\%)$ & $N$ & $(\%)$ & $N$ & $(\%)$ \\
\hline Eating fewer high-fat foods $(N=3284)$ & 1226 & $(66)$ & 679 & $(73)$ & 363 & $(72)$ \\
\hline Eating more fruits and vegetables $(N=3294)$ & 1289 & $(69)$ & 690 & $(74)$ & 376 & $(75)$ \\
\hline Increasing physical activity $(N=3275)$ & 1119 & $(59)$ & 565 & $(60)$ & 316 & (61) \\
\hline Trying to stop smoking ${ }^{e}(N=688)$ & 167 & $(44)$ & 80 & $(40)$ & 56 & $(52)$ \\
\hline Cholesterol checked in previous 5 years $(N=3383)$ & 1173 & $(61)$ & 695 & $(73)$ & 386 & (75) \\
\hline Aspirin use $(N=3383)$ & 366 & (19) & 264 & (28) & 172 & (33) \\
\hline
\end{tabular}

${ }^{a}$ Average risk defined as no first-degree relatives known to have heart disease; total $N=1913$.

${ }^{b}$ Moderate risk defines as one first-degree relative with heart disease; total $N=953$.

${ }^{c}$ High risk defined as two or more first-degree relatives with heart disease; total $N=517$.

${ }^{d}$ Numbers and percentages reflect proportion of respondents in each risk category who answered the corresponding survey question.

${ }^{e}$ Current smokers only.

CVD, cardiovascular disease.

spondents compared with average-risk respondents are included in this report (Table 3). Because the moderate- and high-risk categories contained higher proportions of respondents who reported a personal history of hypertension and hypercholesterolemia, both of which are associated with age and family history, we repeated the analysis using only the 1903 respondents who did not report having hypertension or hypercholesterolemia. For this group of respondents, the association between serum cholesterol measurements and family history-based HD risk was weaker. Moderate-risk respondents were 1.29 (95\% CI 1.03-1.62) times more likely to have a serum cholesterol measured in the previous 5 years than average-risk respondents. High-risk respondents were no more likely than average risk respondents to have a serum cholesterol measured in the previous 5 years (odds ratio $=1.04$; $95 \%$ CI 0.75-1.44). Finally, we compared respondents by whether or not they reported at least one sibling with HD to determine if this had any impact on the practice of CVD risk-reducing behaviors. Aspirin use was the only CVD risk-reducing behavior more likely to be reported by respondents with a sibling who had HD (Table 4).

\section{DISCUSSION}

Among 3383 Healthstyles respondents without a personal history of CVD, nearly one half reported a history of HD in at least one first-degree relative. Overall, more than one half of respondents in this study reported having a serum cholesterol measurement within the previous five years, eating fewer highfat foods, eating more fruits and vegetables, and increasing physical activity to reduce CVD risk. Odds ratios adjusted for age and other demographic variables revealed associations between a moderate or high risk for developing HD based on family history, having serum cholesterol measured within the previous five years and aspirin use to reduce CVD risk. We also observed an association between the report of at least one sibling with $\mathrm{HD}$ and aspirin use to reduce CVD risk.

Table 3

Likelihood of reporting CVD risk-reducing behaviors according to family history-based heart disease risk

\begin{tabular}{|c|c|c|c|}
\hline \multirow[b]{2}{*}{ Behavior } & \multicolumn{3}{|c|}{ Adjusted odds ratio $^{a}$ (95\% Confidence Interval) } \\
\hline & Average risk ${ }^{b}$ & Moderate risk $^{c}$ & High risk $^{d}$ \\
\hline Eating fewer high-fat foods & 1.00 & $1.12(0.94-1.34)$ & $0.97(0.76-1.22)$ \\
\hline Eating more fruits and vegetables & 1.00 & $1.02(0.85-1.23)$ & $0.90(0.74-1.14)$ \\
\hline Increasing physical activity & 1.00 & $0.99(0.84-1.17)$ & $1.12(0.90-1.38)$ \\
\hline Trying to stop smoking ${ }^{e}$ & 1.00 & $0.88(0.61-1.26)$ & $1.43(0.91-2.24)$ \\
\hline Cholesterol checked in previous 5 years & 1.00 & $1.39(1.16-1.67)$ & $1.29(1.01-1.64)$ \\
\hline Aspirin use & 1.00 & $1.49(1.23-1.79)$ & $1.67(1.33-2.09)$ \\
\hline
\end{tabular}

$\overline{{ }^{a} \text { Adjusted for age (continuous), sex, race/ethnicity (white vs. all others), education (some college versus high school or less), income (\$60,000 or more versus }<}$ $\$ 60,000$ ), and whether or not respondent visited a primary care physician within the past year.

${ }^{b}$ Average risk defined as no first-degree relatives known to have heart disease.

${ }^{c}$ Moderate risk defined as one first-degree relative with heart disease.

${ }^{d}$ High risk defined as two or more first-degree relatives with heart disease.

${ }^{e}$ Current smokers only, $N=688$.

CVD, cardiovascular disease. 
Table 4

Likelihood of reporting CVD risk-reducing behaviors among respondents with and without siblings with heart disease

\begin{tabular}{|c|c|c|}
\hline Preventive behavior & $\begin{array}{l}\text { Adjusted } \\
\text { odds ratio }^{a}\end{array}$ & $\begin{array}{c}95 \% \\
\text { Confidence interval }\end{array}$ \\
\hline Eating fewer high-fat foods & 0.84 & $0.63-1.11$ \\
\hline Eating more fruits and vegetables & 1.08 & $0.80-1.45$ \\
\hline Increasing physical activity & 1.02 & $0.79-1.31$ \\
\hline Trying to stop smoking ${ }^{b}$ & 1.66 & $0.95-2.90$ \\
\hline Cholesterol checked in previous 5 years & 0.90 & $0.67-1.20$ \\
\hline Aspirin use & 1.73 & $1.34-2.24$ \\
\hline
\end{tabular}

$\overline{a^{a} \text { Adjusted for age (continuous), sex, race/ethnicity (white versus all others), }}$ education (some college versus high school or less), income ( $\$ 60,000$ or more versus $<\$ 60,000$ ), and whether or not respondent visited a primary care physician within the previous year.

${ }^{b}$ Current smokers only, $N=688$.

CVD, cardiovascular disease.

Because the prevalence of HD increases with age, older respondents might be placed in a higher risk category because they had more first-degree relatives who were already diagnosed with HD. Furthermore, age was associated with both the presence of CVD risk factors (e.g., hypertension and hypercholesterolemia) and the reported practice of CVD risk-reducing behaviors. Because older respondents were more likely to report having a CVD risk factor themselves, they might have been more likely to receive risk-reduction advice from a health care provider. When we controlled for age in both stratified and regression analyses, the associations between family history-based risk and dietary changes were no longer significant. Our findings underscore the need to take age into account when studying family history.

One reason that we did not observe a stronger association between increased family history-based HD risk and the reported practice of risk-reducing behaviors might be that most respondents reported that they engaged in many of the behaviors we studied, regardless of HD risk category. A telephone survey of adults in Olmsted County, Minnesota that used the same series of questions to assess CVD risk-reducing behaviors also found high proportions of participants who were attempting lifestyle changes. ${ }^{16}$ Although $69 \%$ of participants in the Olmsted study reported attempts to change their diet, only $31 \%$ of this group consumed at least five servings of fruits and vegetables per day. Similarly, although $58 \%$ of the participants reported that they were increasing physical activity, only $69 \%$ of this group engaged in daily physical activity. In the Nurses' Health Study, only $3 \%$ of women reported diet, physical activity, and other lifestyle behaviors that placed them in the lowest risk-group for CVD after adjustment for known CVD risk factors, including parental history of myocardial infarction. ${ }^{17} \mathrm{To}-$ gether, these studies suggest that although a large proportion of adults may report attempts to decrease their CVD risk through lifestyle modifications, only a small percentage of individuals are actually practicing these behaviors in a way that could result in a substantial reduction in CVD risk.
Another reason that we did not observe a stronger association between family history-based HD risk and CVD riskreducing behaviors in this study population might be that the respondents did not perceive themselves to be at increased risk for developing HD. HD risk perception was not measured in this study, and we performed the family history-based HD risk classification after the survey data were collected. In a study of individuals at high risk for HD because of a sibling with earlyonset coronary heart disease, unaffected siblings did not perceive their own HD risks to be higher than that of the general population. ${ }^{18}$ A study of adults aged $18-30$ years found that persons who reported a new occurrence of heart attack or stroke in a family member over a 5-year period did not make any sustained lifestyle changes that could reduce their own CVD risk. ${ }^{19}$ However, the authors did not measure perceived risk among this study population and did not provide information to study participants about the implications of a family history of CVD. Another study that assessed the reliability of adult children to report parental deaths from coronary heart disease found that fewer than $30 \%$ of participants with at least one parent who died from coronary heart disease perceived themselves to be at increased risk based on family history. ${ }^{20}$ The findings reported in these three studies suggest that persons who are at increased risk for CVD because of their family history might not recognize their own risk of disease. Interventions that provide education about the significance of family history as a CVD risk factor might motivate persons to make lifestyle changes that could reduce HD risk. ${ }^{9}$

Family history reflects the influence not only of genetics but also of environment and behavior. ${ }^{3}$ Behaviors that can increase CVD risk (e.g., a high-fat diet or sedentary lifestyle) may be more common among the families of the respondents we classified as at increased risk for HD. Therefore, successful family history-based interventions might need to involve entire families in order to produce lasting behavior change. ${ }^{7}$

Investigators have used several different terms to assess family history of coronary heart disease, including heart attack, coronary, and coronary thrombosis.7,21,22 The more general term "heart disease" was used on the Healthstyles survey to assess family history, and the phrase "heart disease or stroke" was used for the questions about CVD risk-reducing behaviors. Persons responding to study questions may understand these terms differently from the meaning intended by the investigators, making it difficult to compare findings from different studies. In this study, we report only the prevalence of a family history of HD and not specifically coronary artery disease because some of the respondents might have included other conditions (e.g., angina pectoris) in their report of affected first-degree relatives.

The findings reported in this study are subject to several limitations. First, we cannot infer causality from our findings because we used cross-sectional data. Second, we relied on self-reported data, which might not provide complete information about respondents' CVD risk-reducing behaviors or family history of HD. In addition, the survey question about family history did not ask about the affected relatives' age at HD onset. However, the preva- 
lences of CVD risk-reducing behaviors and family history of HD among our respondents were similar to those reported by other studies, especially when the high-risk respondents are considered. ${ }^{9,16}$ Furthermore, family history appears to be a valid method for assessment of disease occurrence within families. ${ }^{4}$ In three studies that evaluated the validity of self-reported family history of $\mathrm{HD}$, sensitivity estimates ranged from $67 \%$ to $85 \%$ and specificity estimates ranged from $59 \%$ to $96 \% .7,21,22$ Another limitation of this study is that the sampling methods used for the Healthstyles survey are based on marketing research methods. These methods might generate a sample population different from one that would be selected using traditional epidemiologic methods. Although we cannot generalize the findings in this study to the U.S. population, the Healthstyles survey is based on a national sample. Furthermore, the Healthstyles survey data correlate well with surveillance data on several health conditions, attitudes, and behaviors collected by the Behavioral Risk Factor Surveillance System, a national probability sample telephone survey coordinated by the Centers for Disease Control and Prevention. ${ }^{12}$

The associations we observed between family history-based HD risk, serum cholesterol measurements, and aspirin use to reduce CVD risk might represent interventions suggested by healthcare providers based on individuals' CVD risk factor profiles, including family history, as opposed to self-initiated behaviors. Recognition of the importance of family history as a CVD risk factor might be greater among healthcare providers than among patients. Although taking a family history is an important component of clinical practice, few standards exist that address how this information should be collected, interpreted, and used. In addition, family history is not commonly used to counsel patients about disease risks or preventive behaviors. Our findings underscore the importance of family history and its potential as a tool for risk-based interventions.

We did not observe any significant associations between lifestyle changes (e.g., nutrition, physical activity, and smoking cessation) and family history-based HD risk. Several reports have documented participants' ability to report accurately their family history of HD, but few have examined the association between recognition of the risk associated with a family history of CVD and motivation to change behavior. ${ }^{9,18-20}$ A need exists for research that takes behavioral theory into account to determine if education regarding the importance of family history as a CVD risk factor can be used to motivate behavior change, especially because behavior change can be difficult. ${ }^{23,24}$

Family history-based HD risk classification deserves further study as a means to identify moderate and high-risk individuals who might derive the greatest benefit from interventions designed to reduce CVD risk. ${ }^{9}$ Efforts to develop an effective family history tool for common chronic diseases are currently underway, including a public health research initiative lead by the Centers for Disease Control and Prevention. ${ }^{3,25}$ Research resulting from this initiative may help to answer some of the questions raised by our current study about the role of family history in chronic disease prevention (for more information, see http://www.cdc.gov/genomics/activities/famhx.htm).

\section{ACKNOWLEDGMENTS}

Special thanks to Bill Pollard, Snehal Ruparelia, and Joelyn Tonkin Howard for their assistance with description of the Healthstyles survey and the study population, and to Dr. Julie Magri for her editorial expertise and moral support.

\section{References}

1. Minino AM, Smith BL. Deaths: preliminary data for 2000. National Vital Statistics Reports. Vol 49, no. 12. Hyattsville, Maryland: CDC, National Center for Health Statistics, 2001.

2. American Heart Association. Heart disease and stroke statistics: 2003 Update. Dallas, Texas: American Heart Association, 2002. Available at: http://www.americanheart.org/downloadable/heart/1040391091015HDS_Stats_03.pdf. Accessed February 10, 2003.

3. Yoon PW, Scheuner MT, Peterson-Oehlke KL, Gwinn M, Faucett A, Khoury MJ. Can family history be used as a tool for public health and preventive medicine? Genet Med 2002;4:304-310.

4. Kardia SLR, Modell S, Peyser P. Family-centered approaches to understanding and preventing coronary heart disease. Am J Prev Med 2003;24:143-151.

5. Goldstein LB, Adams R, Becker K, Furberg CD, Gorelick PB, Hademenos G et al. Primary prevention of ischemic stroke: a statement for healthcare professionals from the stroke council of the American Heart Association. Stroke 2001;32:280-299.

6. Liao D, Myers R, Hunt S, Shahar E, Paton C, Burke G et al. Familial history of stroke and stroke risk. Stroke 1997;28:1908-1912.

7. Hunt SC, Williams RR, Barlow GK. A comparison of positive family history definitions for defining risk of future disease. J Chronic Dis 1986;39:809-821.

8. Yoon PW, Scheuner MT, Khoury MJ. Research priorities for evaluating family history in the prevention of common chronic diseases. Am J Prev Med 2003;24:128-135.

9. Hunt SC, Gwinn M, Adams TD. Family history assessment: strategies for prevention of cardiovascular disease. Am J Prev Med 2003;24:136-142.

10. Hahn RA, Heath GW, Chang M-H. Cardiovascular disease risk factors and preventive practices among adults - United States, 1994: a behavioral risk factor atlas. MMWR 1998;47:35-69.

11. Pearson TA, Blair SN, Daniels SR, Eckel RH, Fair JM, Fortmann SP et al. AHA guidelines for primary prevention of cardiovascular disease and stroke: 2002 update. Circulation 2002;106:388-391.

12. Pollard WE. Use of consumer panel survey data for public health communication planning: an evaluation of survey results. In: American Statistical Association 2002 proceedings of the section on health policy statistics; 2002, 2720-2724.

13. Maibach EW, Maxfield A, Ladin K, Slater M. Translating health psychology into effective health communication: the American Healthstyles audience segmentation project. J Health Psych 1996;1:261-77.

14. Scheuner MT, Wang S-J, Raffel LJ, Larabell SK, Rotter JI. Family history: a comprehensive genetic risk assessment method for the chronic conditions of adulthood. Am J Med Genet 1997;71:315-24.

15. Tonkin J. Completeness of family history reporting for asthma and heart disease. [master's thesis]. Atlanta (GA): Emory University; 2002.

16. Thomas RJ, Kottke TE, Brekke MJ, Brekke LN, Brandel CL, Aase LA et al. Attempts at changing dietary and exercise habits to reduce risk of cardiovascular disease: who's doing what in the community? Prev Cardiol 2002:102-108.

17. Stampfer MJ, Hu FB, Manson JE, Rimm EB, Willett WC. Primary Prevention of coronary heart disease in women through diet and lifestyle. N Engl J Med 2000;343:16-22.

18. Becker DM, Levine DM. Risk perception, knowledge and lifestyles in siblings of people with premature coronary disease. Am J Prev Med 1987;3:45-50.

19. Kip KE, McCreath HE, Roseman JM, Hulley SB, Schreiner PJ. Absence of risk factor change in young adults after family heart attack or stroke: the CARDIA study. Am J Prev Med 2002;22:258-266.

20. Watt G, McConnachie A, Upton M, Emslie C, Hunt K. How accurately do adult sons and daughters report and perceive parental deaths from coronary disease? J Epidemiol Community Health 2000;54:859-863.

21. Silberberg JS, Wlodarczyk J, Fryer J, Ray CD, Hensley MJ. Correction for biases in a population-based study of family history and coronary heart disease: the New Castle family history study I. Am J Epidemiol 1998;147:1123-1131.

22. Kee F, Tiret L, Robo JY, Nicaud V, McCrum E, Evans A, Cambien F. Reliability of reported family history of myocardial infarction. BMJ 1993;307:1528-1530.

23. Marteau TM, Lerman C. Genetic risk and behavior change. BMJ 2001;322:10561059.

24. U.S. Preventive Services Task Force. Behavioral counseling in primary care to promote a healthy diet: recommendations and rationale. Am J Prev Med 2003;24:93-100.

25. Yoon PW, Scheuner MT, Khoury MJ. Research priorities for evaluating family history in the prevention of common chronic diseases. Am J Prev Med 2003;24:128-135. 